\title{
NEWS FROM THE INTERNATIONAL FEDERATION OF ROBOTICS
}

1. The International Federation of Robotics is planning to have a World Wide Web presence in the near future. The IFR Web 'pages' ideally will be a first port of call for information about the world robotics scene, including definitions of various types of robots, basic data about the world's robot population, information about international standards regarding robots, addresses of IFR member associations, and details of IFR publications such as the Proceedings of the International Symposia on Robotics.

Already three IFR member associations have established home pages on the World Wide Web. The Robotics Society of Finland has a Web site (in the Finnish language) at http://www.roboyhd.fi/. The Singapore Industrial Automation Association's Web pages can be found at http://www.asia-mfg.com.sg/clients/siaa/ index.html. The US Robotic Industries Association is located on the Web at http://www.robotics.org. An Australian Robot Association Web site is in an advanced stage of preparation.

The Web already contains considerable information about robotics, including details of forthcoming conferences and exhibitions, specifications of commercially available robots and related products such as simulation software and machine vision systems, and news of research activities carried out at government and university laboratories worldwide.

Information about the 27 th International Symposium on Industrial Robots, to be held in Milan from 6 to 8 October and the last Symposium to include the word 'Industrial' in its title, also is available on the World Wide Web at http://muletto.dibe.unige.it/robotics/ robotics.htm.
2. IFR is participating in a new research project on Autonomous Systems. Funded by the Strategic Foundation in Sweden and based at the Royal Institute of Technology in Stockholm, with co-operation from several Swedish industrial partners, the research effort aims to advance robot technology (including sensors, manipulators and software) to make new robot applications possible such as more flexible manufacturing systems, new inspection and repair operations, work in hazardous environments, and personal services.

3. The ISO (International Organization for Standardization) has developed a number of standards for 'manipulating industrial robots operating in a manufacturing environment'. ISO 8373, for instance, establishes terminology (in English and French) for such robots; according to this standard a manipulating industrial robot must be programmable in three or more axes. IFR follows ISO standards when compiling robot statistics. Devices that have only two programmable axes therefore are not included in IFR's annual census of the world's robot population.

However, up to now, no standardised definition has been approved for other types or robots, for example, mobile robots that may operate outside a manufacturing environment and may not necessarily possess a programmable arm. IFR is currently working together with ISO to develop appropriate standards for such robots.

(Submitted by Michael Kassler, Member Executive Committee, International Federation of Robotics.) 